\title{
Internet of Things (IoT) as Green City Economic Development Smart Transportation System
}

\author{
Winardi Slamet ${ }^{1}$, Kamisutara Made ${ }^{2}$, Purworusmiardi Tubagus ${ }^{2}$, Sukoco Agus ${ }^{3}$, and \\ Mudjanarko Sri Wiwoho, * \\ ${ }^{1}$ Computer Science, Computer System Department, Narotama University, Arief Rachman Hakim \\ No. 51, Surabaya, 60117,Indonesia \\ ${ }^{2}$ Computer Science, Informatic System Department, Narotama University, Arief Rachman Hakim \\ No. 51, Surabaya, 60117,Indonesia \\ ${ }^{3}$ Economic \& Business, Management Department, Narotama University, Arief Rachman Hakim \\ No. 51, Surabaya, 60117,Indonesia \\ ${ }^{4}$ Civil Engineering, Civil Department, Narotama University, Arief Rachman Hakim No.51,Surabaya, \\ 60117,Indonesia
}

\begin{abstract}
The number of vehicles in Indonesia based on BPS data in 1987 amounted to 7.98148 million units to 104118969 units in 2013. An increase in the number of vehicles by $1304.51 \%$ is dominated by a motorcycle which was originally $5,554,305$ units to $84,732,652$ units. A large number of vehicles adds to problems of traffic regulation on the road. Internet of Things (IoT) can be used as the vehicle detection control device so as to create solutions Green Economic Development City Smart Transportation System that is good in handling motor vehicle. IoT sensor devices produced can identify the vehicles through model of information system connected with the detection system of motor vehicle license plate identification by means of sensors and data are stored in a digital chip which is mounted on any motor vehicle. With the chip of these vehicles will be produced a Green Economic Development City Smart Transportation System for the development of cities, especially in Narotama.
\end{abstract}

\section{Introduction}

Indonesia is a member of ASEAN which has the largest area, the most populous and has a metropolitan city with the largest number of vehicles. [1] In Java, the most populous island in Indonesia, more than 140 million people live in the region with an area of approximately $130,000 \mathrm{~km} 2$, with a density approaching 1,100 inhabitants per $\mathrm{km} 2$. The high number of demographic and economic growth that followed the increase in urbanization. Population four major cities in Java today has far exceeded millions of lives: Jakarta has nearly 10 million inhabitants, more than 3 million Surabaya, Bandung and Semarang about 2.5 million to 1.5 million residents. This rapid urbanization will continue to increase due to an estimated $60 \%$ of Indonesia's population will live in cities before 2025. The expansion of urban areas and the rapid economic growth resulted in an increased need for movement and transport demand is significant. In fact, urban transport has an

\footnotetext{
*Corresponding author: sri.wiwoho@narotama.ac.id
} 
important economic impact. This condition requires Reduction Strategy Congestion City, which is intended to reduce the burden of congestion in urban areas by strengthening the Management Needs Transportation or a Transportation Demand Management (TDM) with Electronic Road Pricing (ERP), the parking system and the construction of the BRT system, the MRT and improve Traffic Supply Management (TSM) with the development of Intelligent Transportation Systems (ITS). Intelligent Transport Systems (ITS) to regulate the management capacity and priorities so as to create an environmentally friendly city.

[10] Indonesia has a population of 248818100 people, an area of $1,910,931.32 \mathrm{~km}^{2}$ and the number of motor vehicles as 104.118.969unit. Statistics Indonesia (2017),

the growth of motor vehicles in July 2016 reached 124348224 units consist of private cars, motorcycles, buses, and trucks. Growth potential vehicle traffic congestion and the potential for traffic violations are also getting bigger. Limited number of traffic officers will have difficulty in monitoring and vehicle settings. The composition of the number of traffic officers and the number of vehicles is not impartial. Solving these problems can be reduced using components such as digital chip attached on the vehicle license plate. Identification of any motor vehicle digitally facilitate supervision and regulation by traffic officers. Digital chip contains the identity of the motor vehicle and emitted continuously while in a highway through a wifi signal. The test was conducted on a digital chip limited area of Narotama, Surabaya, Indonesia.

\section{System Description \\ 2.1 Internet of Things (IOT)}

Internet of Things (IOT) means "an open network of devices or things that are uniquely addressed based on standard communication protocols", the which is a novel prototype that is rapidly gaining ground in the scenario of modern wireless communications. The basic idea here is that the Omnipresence of a multifarious things or objects such as RF tags, sensors, actuators, mobile devices / things, etc are Able to interaction with each other to Obtain common goals through unique schemes of inscribing. The flairs offered by the IOT help in flourishing un-hackneyed protocols in varied domains (1) Traffic transportation and Logistics domain. (2) Healthcare domain (3) Smart environment (home, office, plant) domains. (4) Private and Social domains.

\subsection{Positioning}

A GPS consists of three distinct parts as it is embellished in the following Figure 1. Reviews These three parts are: the satellites in orbit, the ground control stations, and the users (satellite receivers found in land, air, sea). For the part of satellites in orbit, out of the twenty four, twenty-one are in operation. 21 Among These four satellites are visible at any time from any station on earth. The latitude and longitudinal position for each specific station is feasible to be Obtained in the form of ' $x$ ', ' $y$ ' and ' $z$ ' coordinates (position vector). The information concerning the speed $(\mathrm{dx} / \mathrm{dt}, \mathrm{dy} / \mathrm{dt}, \mathrm{dz} / \mathrm{dt})$ of a vehicle IS ALSO available all over the world, at any time, and under all weather conditions. Ground control station contains a master control station, monitoring station and injecting station. The master manipulates the satellite ephemeris and correction criterion of satellite clock, and injection of the data into the Reviews These satellites. Moreover it controls the satellite and issues Ordinance to it as well as associates in monitoring. Monitoring station perceives the reception of satellite signals; superintending the working status of satellites; injecting station effectuates the injection of satellite ephemeris and clock correction parameters of satellite. 


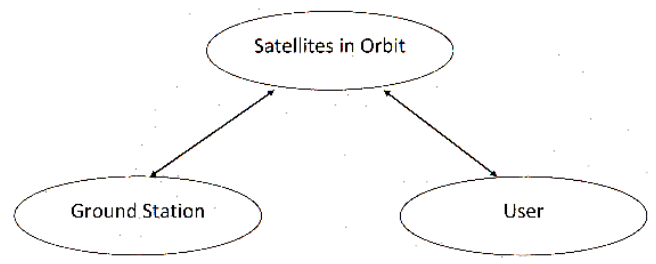

Fig. 1. Parts of GPS

\subsection{Green Transport}

[2] Green transport is sustainable transport concept is the use of environmentally friendly technology in an effort to meet the transportation needs of the community. In the context of urban planning, this concept is translated as an effort to improve community facilities for bikes, pedestrians, communication facilities, as well as mass public transport provision inexpensive and environmentally friendly such as electric trains and other public transportation to reduce private vehicle. [6] They are people, ideas, networks, knowledge, planning and visions. Later in the paper they will be illustrated, showing how they connect and interaction in the proposed draft strategy for Cluj-Napoca Romania. A smart city is a connected city. Just like the cases Analyzed in the previous pages, the which both located in strategic locations and poses an extremely well developed and advanced Infrastructures, representing around mobility hubs in their area. That is also available for Cluj-Napoca, a city which is said to be located in a advantaging position.

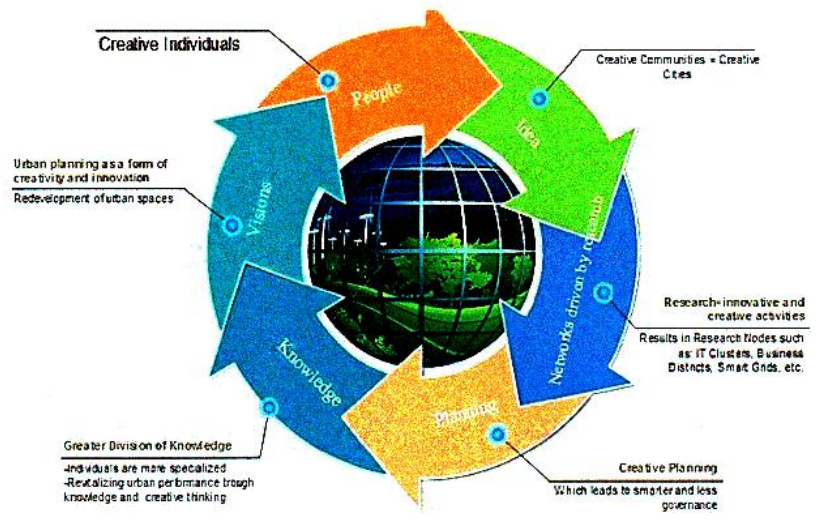

Fig.2. Conceptual Elements of Smart City. Own Sources [8]

[11] Center is a state of not moving a motor vehicle or motor can be the start of a journey to a specific period in accordance with the circumstances and needs require an area as stops were organized by the government and other parties who may be individuals or business entity. Various patterns are applied management in several cities in Indonesia and even in other countries, including in Malaysia. Parking problems do occur in various countries around the World. Parking management are applied starting with the conventional management and use of the latest management in the form of digital infrastructure. In the Klang Valley, vehicle ownership is an exceptional factor and plays a significant role in the Influencing parking demand as there are around 500,000 new vehicle registrations added to the region every year [5]. Also Malaysia tops the list as the country with the third highest car ownership in 2014, 88\% of Malaysian car Also owners intend to upgrade Reviews their 
vehicle when they are financially-able [8]. With car ownership in the growing trend, the parking demand in the Klang Valley, especially at rail stations, is expected to increase is.

\subsection{Management Needs Transport}

[12] The elements of the transportation demand management (Transportation Demand Management (TDM)) consists of:

1. Restricting access to cars by improving public transport services and provide an incentive for users commuters.

2. Reduce car mobility reallocation of road space and limited traffic zones by improving public transport services, improving infrastructure buses, bicycles, pedestrians and improving mobility options

3. Land use planning are integrated with non-motorized transport planning

4. Repression / law enforcement through awareness-raising community awareness of traffic

\subsection{Vehicle Identification Numbers}

In the Law of the Republic of Indonesia Number 22 Year 2009 regarding traffic and road transport regulates TNKB as vehicle registration and vehicle identity to be registered. In article 64 to article 70 and article 78 regulates the process of registration and identification of vehicles and issued BPKB, vehicle registration, and TNKB as proof of identity of the motor vehicle. The shape of the motor vehicle identity issued by the police are as follows:

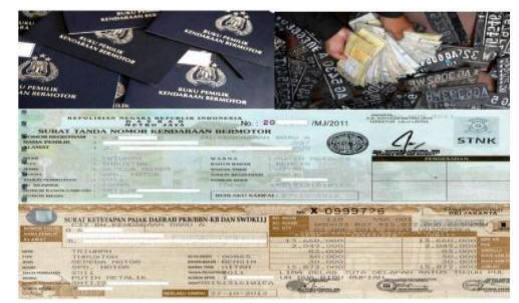

Fig.3 Form BPKB, vehicle registration, and TNKB

Such deficiencies should be owned by every owner of a motor vehicle, when driving a motor vehicle shall be equipped TNKB attached to the motor vehicle at the front and rear of a motor vehicle and a driver must carry vehicle registration as proof of driving a motor vehicle in accordance with Government Regulation No. 80 of 2012 and a driver's license as the identity of a driver who has been authorized by the police for driving a motor vehicle on the road in accordance with the type of vehicle. Installation TNKB vehicles conforming to the standards is as follows:

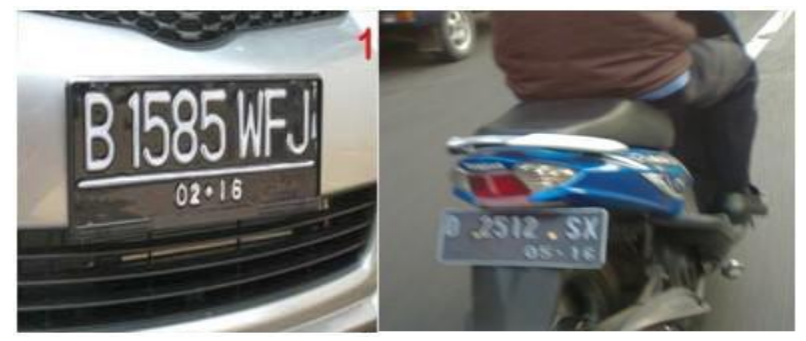

Fig.4. Installation Number Plate (TNKB) 


\section{Methodology}

\subsection{Vehicle Identification Numbers}

Hardware Design of Digital Vehicle Identity

Identification of the vehicles were built using a chip ESP8266 provides vehicle data and the owners are always transmitted continuously via a wifi signal.

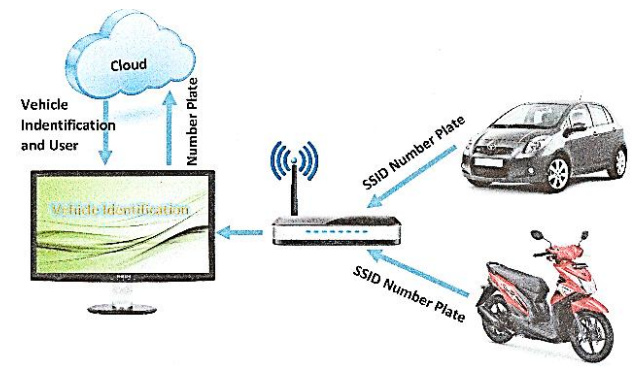

Fig.5. System Block Diagram

Every motor vehicle both cars and motorcycles are all given a chip that contains the identity of the vehicle as listed in the vehicle registration date. The chips are used to store the identity of a motor vehicle using the ESP8266. This chip will be given identity (SSID) in the form of any motor vehicle license plate number because it is unique (different every motor vehicle).

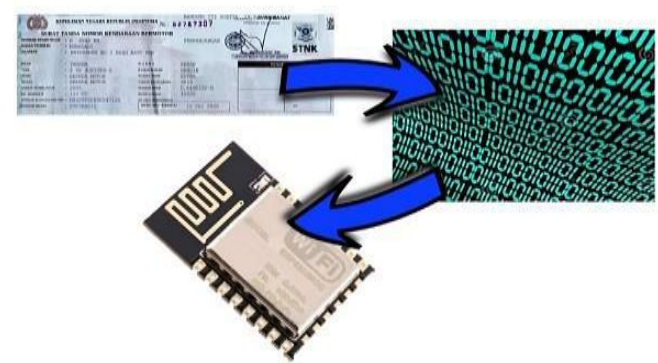

Fig.6. Embedded Chip Identification of Vehicles to ESP8266

Once all the data entered ESP8266 paired subsequent chip in a motor vehicle and serves as a client that transmits data. If found access point then this chip will connect and provide access point SSID to subsequently forwarded to the server to be recorded in the vehicle identification data base. Each client will have an IP that is obtained from the configuration after connecting with the wifi signal at the access point. Computer and chip ESP8266 must be connected to the same access point. Once the chip is filled ESP8266 next vehicle identification chip is mounted on a motor vehicle in accordance with the license plate number.

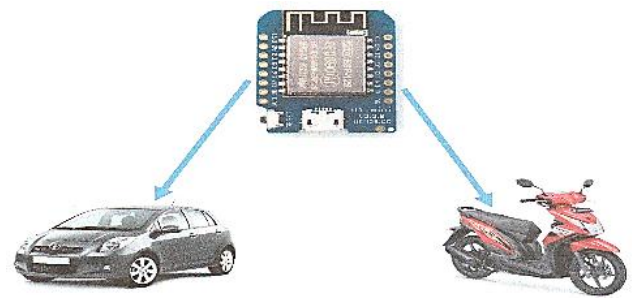

Fig.7. Vehicle Identification Digitally 


\subsection{Data Flow Analysis}

\section{Design Software}

To realize this motor vehicle identification required two software namely Arduino IDE and PHP. ESP8266 chip is programmed using the Arduino IDE and Server using PHP. Arduino software to design hardware for the client and the router while PHP for the purpose of designing the web service.

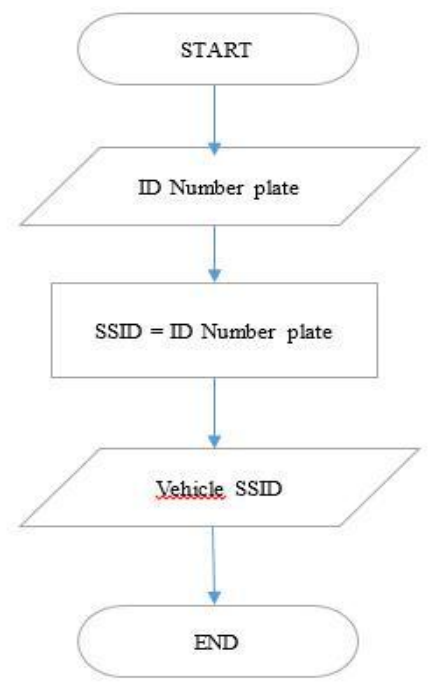

Fig.8. Flowchart Client

For client hardware mounted on a motorcycle or car in the form of chips ESP8266 with a program filled in the form of police numbers are used as SSID (Service Set Identifier) using the Arduino IDE and emitted continuously by a wifi signal.

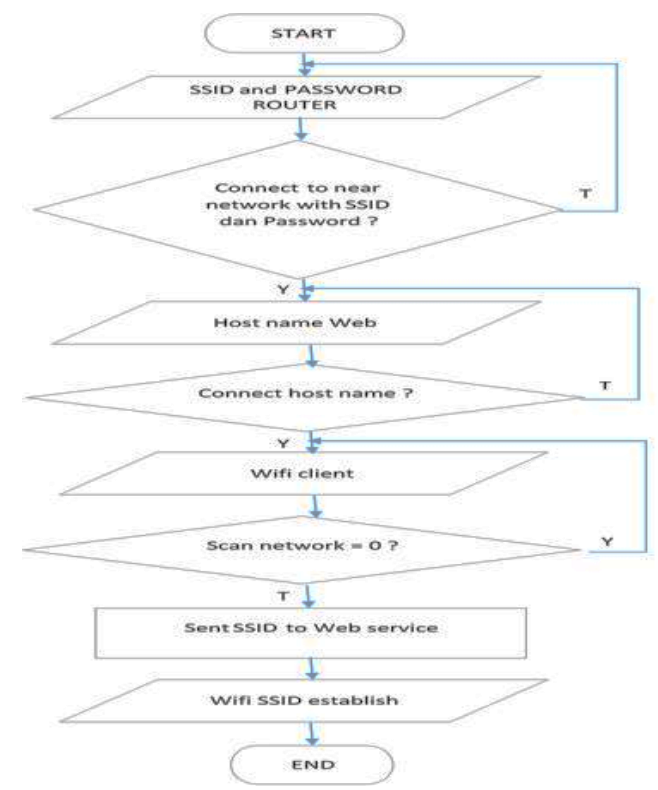

Fig.9. Flowchart Router 
In this router also uses the chip ESP8266 filled program to detect the presence of a motor vehicle by capturing SSID motor vehicle emitted through a wifi signal. This router must first be connected to the Internet network via the access point nearby. The domain name system, or commonly abbreviated as DNS is a system that serves to translate IP addresses into domain names and vice versa, from domain names to IP addresses. Thus, the host computer sends queries in the form of a computer name and domain name server which is then mapped into an IP address by the DNS. After getting the IP and the next router will scan the wifi signal from the client (SSID motor vehicles) whether there are vehicles which are located around the router, If any SSID motor vehicle router will send the SSID to the server (web service) then the web server will bring the SSID the.

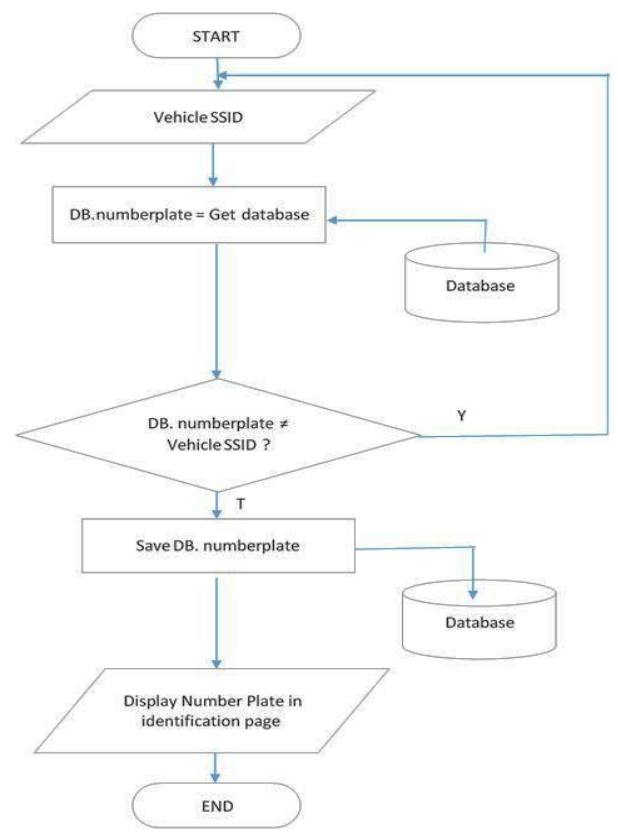

Fig.10. Flowchart Web Service

Once the router sends the SSID of the motor vehicle to the web service next system will check the database if the SSID is already registered or not, if not it will display the license plate number of the vehicle only, while when it is registered with the web service will show complete data motor vehicle besarta the identity of the vehicle owner motorized. The existence of the motor vehicle will be stored in the database and displayed on the monitor screen.

\section{Results Analysis and Discussion}

After designing the hardware client and the router using Wemos ESP8266 and identity database servers to accommodate motor vehicles. Routers are placed around the parking area off parking the motorcycle and the car park for the wifi signal range of about 10 meters. This router will capture client wifi signal in the form of motor vehicle setup SSID form plate number (license plate number). Then the router will forward the SSID to the data server and then be displayed on the monitor screen in the form of the identity of the motor vehicle. 


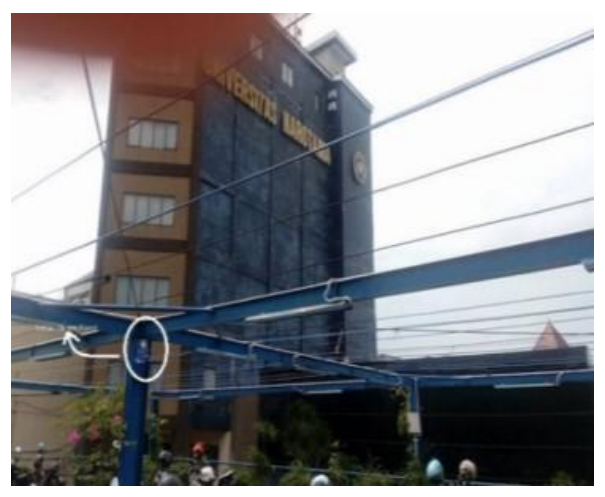

Fig.11. Router Installation

ESP8266 chips that function as a client filled by the identity of the motor vehicle in the form of police numbers, then the chip would emit wifi signal continuously until it was discovered by a router. In this case the identity of the motor vehicle license number L 6125 QZ police, license plate number is used as the SSID name of the client. Every motor vehicle shall have a different SSID for using the identification number issued by the authorities, namely the police, and indeed any motor vehicle license number will be different.
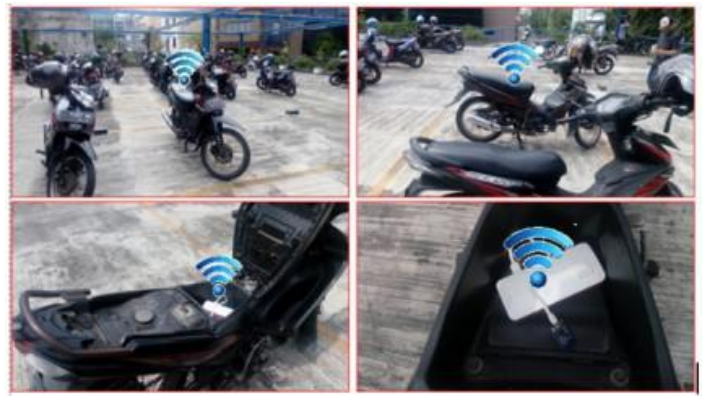

Fig.12. Identification Motorcycles

As with motorcycles, cars were given chip ESP8266 who has filled the car's identity .. Identification of complete vehicles and vehicle owners are stored in the database server. In places parker Narotama University campus area in pairs of a router to identify vehicles that are around the router. The router will always scan wifi signals are scattered around, but the only format number plate only to be captured by the router and forwarded to the server to display the identity of a motor vehicle on the screen. This router will automatically send data to the server when the client has a wifi signal formats such as vehicle license plate. In this case use xenia car with police number L $1676 \mathrm{HB}$ in parking near university mosque Narotama. 

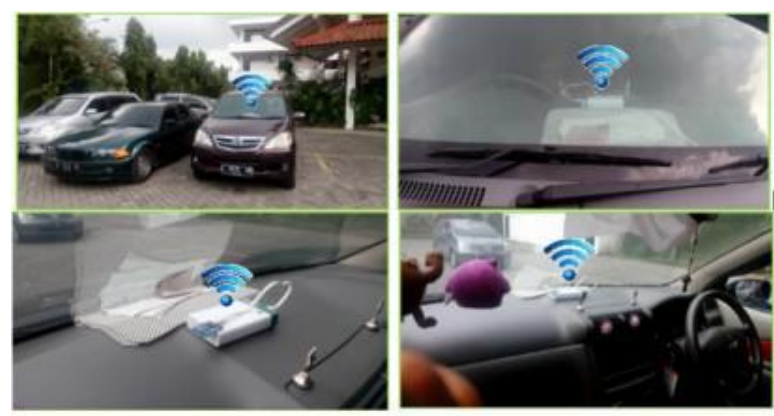

Fig.13. Identification Car

Regular users of motor vehicles to the campus of the University Narotama obliged to register the identity of the vehicle to a server computer to be recorded in the database of vehicle owners. Entitled to enter the motor vehicle identity data is the admin of this system. If you are already registered then the system will display the data of the motor vehicle owner, if a motor vehicle license plate will be displayed any time and date and hours of admission to college.

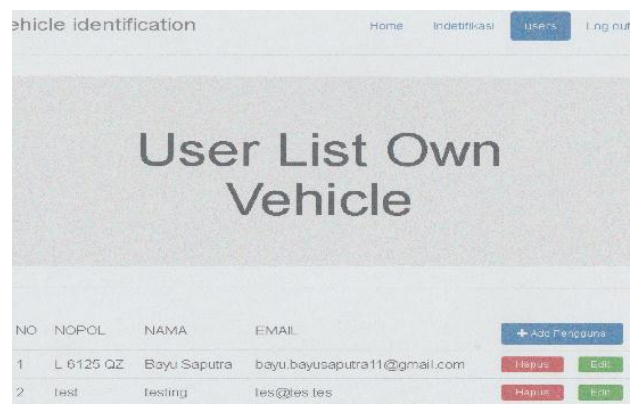

Fig.14. Database Own Vehicle

Vehicle data has been embedded in the chip ESP8266 in the motor vehicle will be recognized by a router that has been installed at certain places that can reach the existence of a motor vehicle. The signals emitted by the client will be caught by the next router and client SSID will be forwarded to the data server to retrieve the database that was inputted in its own server and will be displayed in a monitor to detect vehicles which are located around the router. For the time being displayed in this system is the last number of police and he is connected with the router. Data can be developed so that is displayed in the system more complete.

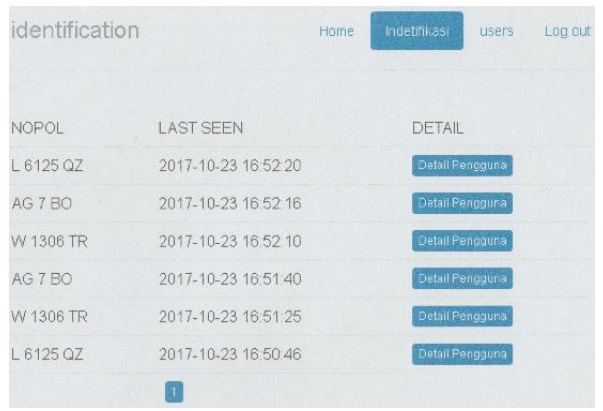

Fig.15. List Vehicle Identified 


\section{Conclusion}

The conclusion of the identification system in the form of a motor vehicle with wifi technology

1. The chip is able to identify license plates with the code specified by the police of the Republic of Indonesia.

2. The chip detection can be developed in other countries based on their vehicle license and transportation system standards.

3. The chip can detect the presence of vehicles not more than 100 meters in distance by using wifi signal emitted by those vehicles.

4. This digital identification system can help identifying vehicles in case of crucial problems.

5. This digital identification system can help government programs in transportation, for examples restriction program on vehicles uses on highways using the rules of odd and even number plate and the restriction program on fuel purchases

\section{References}

1. AE. Andersson, C. Mellander, Handbook of Creative Cities, Edwar Elgar Publishing Limited, UK, (2011)

2. B. Ganesh, Harisudha, Efficient Transportation System Using the Internet of Things, the International Journal of Applied Engineering Research, ISSN 0973-4562 510 pp. 3979-3982, (2015)

3. D. Gusnita, Green Transport: Transport and Sustainable Contribution to Reduce Pollution Air, Research Center of Atmospheric Sciences and Climate, LAPAN, Aerospace News 11, pp. 66-71, 6 (2010)

4. F. Kawsar, D. Fitton, and V. Sundramoorthy, Smart objects as building blocks for the internet of things, Internet Computing, IEEE 14, pp. 44-51, (2010)

5. Government Transformation Program, Improving Urban Public Transport, National Key Results Areas, GTP Annual Report 2014, Performance Management and DeliveryUnit (GUIDE), (2014)

6. H. Phooi, Future Parking Demand at Rail Stations in the Klang Valley, ISCEE pp. 103.09001 (2017)

7. J. Lee, J. Jeong, T. Oh, J. Jun and SH. Son, DCMAC: Data-Oriented Cluster-Based Media Access Control Protocol for Vehicular Networks, 30th Int Conf on Advanced Information Networking and Applications workshops (WAINA), Crans-Montana, pp. 258-261 (2016)

8. J. Supriono, SW. Mudjanarko, Performance Evaluation Parking In RSU Haji Surabaya,. E-Journal Spirit Pro Patria, (2016)

9. Ministry PUPR, Statistical Information Infrastructure, Public Works and Public Housing (BIS-PUPR), Statistics Indonesia, pp : 34 (2015)

10. R. Nielsen, Middle Class Will Drive Demand in the Global Automotive ComingTwo Years, (2014)

11. T. Rye, Parking Management: A Contribution to the city livable, Deutsche Gesellschaft Fur Internationale Zusammernabeit (GIZ) GmbH, Federal Ministry For Economic Cooperation and Development (BMZ), (2010)

12. The Agence Française de Développement, Finding a solution to urban transport: the challenge of financing and network integration 6 (2014) 